\title{
Comportamento fluência-recuperação de ligantes asfálticos modificados com copolímero SBR e ácido polifosfórico
}

\author{
Matheus David Inocente Domingos ${ }^{1}$, Adalberto Leandro Faxina ${ }^{2}$
}

\begin{abstract}
Resumo: Este trabalho avaliou os comportamentos fluência-recuperação de ligantes asfálticos modificados com o copolímero SBR (CAP+SBR), ácido polifosfórico (CAP+PPA) e uma combinação de ambos (CAP+SBR+PPA) por meio do ensaio de fluência e de recuperação sob tensão múltipla (MSCR) em temperaturas entre 52 e $76^{\circ} \mathrm{C}$. Todas as formulações apresentam a mesma classificação em temperaturas altas (PG 76-xx) e o CAP 50/70 é classificado como PG 64-xx. Aumentos no percentual de recuperação $(\mathrm{R})$ e reduções na compliância não-recuperável $\left(\mathrm{J}_{\mathrm{nr}}\right)$ foram observados após os processos de modificação, o que é favorável à resistência do CAP à deformação permanente. Os níveis recomendados de tráfego se mostraram semelhantes para o $\mathrm{CAP}+\mathrm{SBR}+\mathrm{PPA}$ e para o CAP+PPA, sendo também maiores do que aqueles encontrados para o $\mathrm{CAP}+\mathrm{SBR}$. Isto indica que o PPA tem potencial para substituir o uso do SBR na modificação do CAP, desde que a formulação não apresente uma suscetibilidade excessiva ao trincamento por fadiga e ao trincamento térmico.

Palavras-chave: Ligantes asfálticos modificados, percentual de recuperação, compliância não-recuperável, copolímero SBR, ácido polifosfórico, grau de desempenho.
\end{abstract}

\begin{abstract}
The creep-recovery behavior of asphalt binders/cements modified with SBR copolymer (AC+SBR), polyphosphoric acid (AC+PPA) and a combination of both (AC+SBR+PPA) at temperatures from 52 to $76^{\circ} \mathrm{C}$ was analyzed by means of the multiple stress creep and recovery (MSCR) test. All the formulations have the same high PG grade (PG 76$\mathrm{xx}$ ) and the 50/70 base binder is graded as PG 64-xx. Increases in the percent recovery (R) and decreases in the nonrecoverable compliance $\left(\mathrm{J}_{\mathrm{nr}}\right)$ were observed after the modification process, which is favorable to the AC resistance to rutting. The $\mathrm{AC}+\mathrm{SBR}+\mathrm{PPA}$ and the AC+PPA showed similar acceptable traffic levels within the whole temperature range, and they were higher than the ones obtained for the AC+SBR. This indicates that PPA may be an alternative to replace the SBR copolymer on asphalt cement modification, provided that the formulation is not highly susceptible to fatigue cracking and thermal cracking.
\end{abstract}

Keywords: Modified asphalt binders, percent recovery, nonrecoverable compliance, SBR copolymer, polyphosphoric acid, performance grade.

\section{INTRODUÇÃO}

Um dos principais mecanismos de ruptura dos pavimentos rodoviários consiste no acúmulo de deformações plásticas - também chamadas de permanentes - nas faixas de tráfego por ocasião das passagens das cargas dos veículos. Este mecanismo de ruptura, designado como deformação permanente ou rutting na nomenclatura norteamericana, pode ser combatido por meio de medidas como através da compactação eficiente da mistura asfáltica dentro dos limites especificados em projeto, uma escolha consciente dos agregados e da granulometria e a elaboração de um projeto estrutural que limite as tensões atuantes no subleito a intervalos de valores admissíveis e seguros. Pode-se também adicionar modificadores ao ligante asfáltico de base (Cimento Asfáltico de Petróleo - CAP), fazendo com que a resistência da mistura asfáltica à deformação permanente seja ainda maior. Estes modificadores abrangem desde os menos conhecidos como os antioxidantes e as fibras de poliéster e de polipropileno até os mais conhecidos como a borracha moída de pneus, o copolímero de estireno-butadieno-estireno (SBS) e a borracha de

\footnotetext{
${ }^{1}$ Matheus David Inocente Domingos, Departamento de Engenharia de Transportes, Escola de Engenharia de São Carlos, Universidade de São Paulo. (matheusdavid@sc.usp.br)

${ }^{2}$ Adalberto Leandro Faxina, Departamento de Engenharia de

Transportes, Escola de Engenharia de São Carlos, Universidade de São

Paulo. (alfaxina@sc.usp.br)

Manuscrito recebido em 08/06/2014 e aprovado para publicação em 24/03/2015.

Este artigo é parte de TRANSPORTES v. 23 , n. 2, 2015. ISSN: 2237-1346 (online). DOI:10.14295/transportes.v23i2.800.
}

estireno-butadieno (SBR).

O copolímero SBR é formado por blocos de poliestireno e de polibutadieno, assim como o copolímero SBS. Uma diferença entre os dois modificadores está na distribuição dos blocos na cadeia polimérica: esta distribuição é do tipo ordenada no SBS - um bloco de poliestireno quimicamente combinado com um bloco de polibutadieno e com outro de poliestireno - e do tipo aleatória no SBR (King et al., 1999; Leite, 1999). O SBR é geralmente incorporado ao CAP na forma de dispersão em água, isto é, no formato de emulsão. As partículas de borracha são extremamente pequenas e uniformes quando estão em emulsão e, ao serem misturadas ao CAP, dispersam-se rapidamente por todo o material, formando uma estrutura reticulada reforçada (Yildirim, 2007). A adição de SBR ao CAP é rápida e não requer agitação de alto cisalhamento. Esta adição provoca aumento do módulo complexo e aumento do ponto de amolecimento do material, além da possibilidade de obtenção de ligantes asfálticos com graus de desempenho da mesma ordem de grandeza encontrada em formulações com SBS (Leite, 1999).

Além de aumentos no módulo complexo e no grau de desempenho da classificação Superpave ${ }^{\circledR}$, a literatura técnica destaca ainda outras vantagens com relação a modificação do CAP com o copolímero SBR. Estas vantagens incluem o aumento da ductilidade do material em temperaturas baixas, o aumento da recuperação elástica, a melhoria na coesão e na adesão do ligante asfáltico aos agregados e a redução da taxa de oxidação (Yildirim, 2007; Zhang et al., 2007; Zhang et al., 2009; Zhang e Yu, 2010). Entretanto, formulações CAP+SBR podem apresentar problemas relacionados à estabilidade à estocagem quando 
o copolímero não é adicionado na forma de emulsão. Em tais casos, a incorporação de outros modificadores como os compostos à base de enxofre e/ou ácido polifosfórico (PPA) podem melhorar a compatibilidade entre o SBR e o CAP de base (Zhang e Yu, 2010).

Como já destacado, o PPA também pode ser utilizado como um modificador do ligante asfáltico. Especificamente, o PPA é um oligômero produzido por meio da condensação do ácido monofosfórico ou da hidratação do pentóxido de fósforo $\left(\mathrm{P}_{2} \mathrm{O}_{5}\right)$. Este ácido é do tipo médioforte e não possui água livre em sua composição, além de ser um líquido viscoso à temperatura ambiente de $25^{\circ} \mathrm{C}$ (Masson, 2008; Baumgardner, 2012). O PPA é altamente solúvel em compostos orgânicos como o CAP e, quando diluído em água, transforma-se lentamente em ácido ortofosfórico. O teor de PPA e os efeitos da sua adição no CAP dependem da composição química do material de base, o que está relacionado ao petróleo de origem (Baumgardner, 2012). A presença do PPA permite a redução do teor de polímero na formulação e auxilia o material modificado no cumprimento dos critérios da especificação Superpave ${ }^{\circledR}$, tais como os limites mínimos do parâmetro $\mathrm{G}^{*} / \mathrm{sen} \delta(1,0$ e $2,2 \mathrm{kPa})$, o limite máximo do parâmetro $\mathrm{G}^{*} \operatorname{sen} \delta(5.000 \mathrm{kPa})$ e a viscosidade rotacional máxima de 3,0 Pa.s a $135^{\circ} \mathrm{C}$ (Buncher e D’Angelo, 2012).

O ensaio de fluência e de recuperação sob tensão múltipla, mais conhecido como MSCR (sigla no inglês para multiple stress creep and recovery), é um ensaio relativamente recente para avaliação da suscetibilidade do CAP à deformação permanente. Desenvolvido pela Federal Highway Administration (FHWA) dos Estados Unidos, a partir de um refinamento do ensaio de fluência repetida e de recuperação (D’Angelo et al., 2007), o MSCR possui um protocolo de ensaio simples e os parâmetros resultantes são fáceis de serem calculados. Este protocolo consiste basicamente na aplicação de ciclos sucessivos de carregamento (creep) e de recuperação (recovery) em uma amostra de ligante asfáltico condicionada entre duas placas paralelas de $25 \mathrm{~mm}$ de diâmetro ensaiada no DSR e no monitoramento das deformações do material em cada ciclo. As correlações entre os resultados do MSCR e as deformações permanentes de misturas asfálticas ensaiadas em campo ou em laboratório, avaliadas por meio do coeficiente de determinação $R^{2}$, têm se mostrado altas - iguais ou superiores a 0,70 - em diversos estudos científicos (D'Angelo et al., 2007; D'Angelo, 2009; Dreessen et al., 2009; Reinke, 2010; Anderson, 2011; Martins et al., 2011; Anderson e Bukowski, 2012; Gierhart, 2013). Como consequência direta destes resultados, o MSCR foi incorporado à nova versão da especificação Superpave ${ }^{\circledR}$ e o parâmetro $\mathrm{J}_{\mathrm{nr}}$ (compliância não-recuperável) foi escolhido como o novo indicador da suscetibilidade do CAP à deformação permanente (AASHTO MP19, 2010).

O outro parâmetro reológico que pode ser extraído do ensaio MSCR, designado como percentual de recuperação (R), mede diretamente a resposta elástica do CAP e tem se mostrado capaz de estimar a estrutura e a extensão da rede polimérica na formulação (D’Angelo, 2010). Pesquisas envolvendo ligantes asfálticos modificados do tipo $\mathrm{CAP}+$ modificador+PPA e CAP+modificador sugerem que os percentuais de recuperação podem apresentar diferenças significativas de uma formulação para outra - desde 14 até $244 \%$ no caso dos trabalhos de D’Angelo e Dongré (2009) e D'Angelo (2010) - e que o ensaio MSCR é indicado para distinguir as respostas reológicas das formulações com e sem polímeros (Fee et al., 2010). Embora o percentual de recuperação não seja oficialmente utilizado na especificação Superpave ${ }^{\circledR}$, seu valor pode ser empregado juntamente com a compliância não-recuperável do ligante asfáltico para avaliar o nível de elasticidade - alta ou baixa - da formulação, desde que esta compliância seja igual ou inferior a 2,0 kPa. (Anderson, 2011; Anderson e Bukowski, 2012).

Tendo como referência as inovações conquistadas com o desenvolvimento do MSCR no campo da reologia dos ligantes asfálticos e o uso cada vez maior de ligantes asfálticos modificados em obras de pavimentação, o objetivo principal deste trabalho é verificar a viabilidade da substituição (total ou parcial) do copolímero SBR pelo PPA na modificação de um CAP de base 50/70, considerando formulações de mesmo grau de desempenho em temperaturas altas (PG 76-xx) e apenas o mecanismo de ruptura por deformação permanente. Esta análise foi feita em temperaturas entre 52 e $76{ }^{\circ} \mathrm{C}$ e nos dois níveis de tensão padronizados para o ensaio MSCR (100 e 3.200 $\mathrm{Pa})$, para materiais envelhecidos a curto prazo.

\section{PROGRAMA EXPERIMENTAL}

Para a preparação dos ligantes asfálticos modificados com PPA, SBR e SBR+PPA, foram selecionados os seguintes materiais: (a) um CAP 50/70 de classificação por grau de desempenho PG 64S-xx, com grau PG contínuo de $67^{\circ} \mathrm{C}$ e com perda de massa de $-0,1094 \%$, fornecido pela refinaria Replan-Petrobras de Paulínia-SP; (b) o copolímero SBR de designação comercial Solprene ${ }^{\circledR} 1205$, fornecido pela Dynasol Elastomers; e (c) o ácido polifosfórico (PPA) de designação comercial Innovalt ${ }^{\circledR}$ E200, fornecido pela Innophos Inc. dos Estados Unidos. Este copolímero SBR apresenta um teor de estireno de $25 \%$, dos quais $17,5 \%$ estão presentes na forma de blocos de poliestireno. A seleção dos teores de cada modificador foi feita de tal maneira que o CAP+PPA, o CAP+SBR e o $\mathrm{CAP}+\mathrm{SBR}+\mathrm{PPA}$ apresentam o mesmo grau de desempenho em temperaturas altas (PG 76-Xx) na nova classificação Superpave ${ }^{\circledR}$ para ligantes asfálticos, dada pela norma AASHTO MP19 (2010). Segundo esta classificação, a temperatura alta do PG do CAP é aquela na qual o parâmetro $\mathrm{G}^{*} / \mathrm{sen} \delta$ possui um valor mínimo de $1,0 \mathrm{kPa}$ na condição virgem. Todos os ligantes asfálticos modificados foram preparados em um misturador de baixo cisalhamento da marca Fisatom, modelo 722D. Outras informações sobre as formulações podem ser conferidas na Tabela 1 .

Os seguintes ensaios foram selecionados para caracterizar determinadas propriedades reológicas dos ligantes asfálticos nas condições virgem e envelhecida a curto prazo: penetração a $25^{\circ} \mathrm{C}$ (ASTM D5, 2006), ponto de amolecimento (ASTM D36, 2009) e viscosidade rotacional a $135^{\circ} \mathrm{C}$ (ASTM D4402, 2006). Os ensaios de penetração foram conduzidos em um penetrômetro universal da marca Solotest e os valores finais, que foram empregados no cálculo da penetração retida $\mathrm{R}_{\mathrm{PEN}}$, representam a média de quatro medições individuais para cada material. Os ensaios de ponto de amolecimento foram realizados em um equipamento automático da marca ISL e modelo RB $36-5 \mathrm{G}$ e os valores finais, que foram utilizados no cálculo do aumento do ponto de amolecimento $\mathrm{I}_{\mathrm{R} \& \mathrm{~B}}$, também cor- 
Tabela 1. Informações técnicas sobre as formulações e resultados de perda de massa

\begin{tabular}{llcc}
\hline descrição do item & $\boldsymbol{C A P + P P A}$ & $\boldsymbol{C A P + S B R}$ & $\boldsymbol{C A P + S B R + P P A}$ \\
\hline classificação PG (AASHTO MP19, 2010) & $76 \mathrm{~S}-\mathrm{xx}$ & $76-\mathrm{xx}$ & $76 \mathrm{~S}-\mathrm{xx}$ \\
grau PG contínuo $\left({ }^{\circ} \mathrm{C}\right)$ & 77,8 & 76,6 & 81,3 \\
teor de ligante asfáltico (\% em massa) & 98,8 & 94,5 & 96,0 \\
teor de copolímero SBR (\% em massa) & - & 5,5 & 3,5 \\
teor de PPA (\% em massa) & 1,2 & - & 0,5 \\
temperatura de mistura $\left({ }^{\circ} \mathrm{C}\right)$ & 130 & 180 & 180 \\
tempo de mistura (min) & 30 & 120 & $120^{*}$ \\
velocidade de rotação (rpm) & 300 & 400 & 300 \\
perda de massa $(\%)$ & $-0,2263$ & $-0,0815$ & $-0,2499$ \\
\hline
\end{tabular}

* O ácido polifosfórico (PPA) foi adicionado à formulação CAP+SBR após 90 min de mistura.

respondem à média de quatro medições individuais para cada ligante asfáltico.

As viscosidades rotacionais, referentes à média de duas medições para cada material e condição de envelhecimento, foram obtidas em um viscosímetro rotacional da marca Brookfield e modelo DV-II+Pro, acoplado a um controlador de temperatura Thermosel. O spindle 21 foi empregado nestas medições de viscosidade, e a velocidade rotacional foi escolhida de maneira a manter a porcentagem de torque entre 10 e $98 \%$ da capacidade máxima do equipamento, conforme estipulado na norma ASTM D4402 (2006). Ao final, os índices de aumento da viscosidade $\left(R_{\mathrm{V}}\right)$ - razão entre as viscosidades envelhecida e virgem - foram calculados.

O envelhecimento dos ligantes asfálticos a curto prazo seguiu os procedimentos estabelecidos na norma ASTM D2872 (2004). Após o ensaio, as amostras foram então retiradas e suas massas antes e após o envelhecimento foram utilizadas nos cálculos da perda de massa $\left(\mathrm{M}_{\mathrm{L}}\right)$. Resultados negativos de $\mathrm{M}_{\mathrm{L}}$, tais como os obtidos nesta pesquisa (Tabela 1), indicam que os efeitos da volatilização foram mais intensos do que os efeitos da oxidação. Em outras palavras, a perda das frações leves do CAP foi maior do que o ganho de massa por conta da oxidação durante o envelhecimento. As maiores perdas de massa podem ser encontradas no CAP+SBR+PPA $(-0,2499 \%)$ e no CAP+PPA $(-0,2263 \%)$, seguido pelo CAP puro ($0,1094 \%)$ e finalmente pelo CAP+SBR (-0,0815\%). Estas observações permitem dizer que alguns tipos de modificação são capazes de reduzir a perda de massa do ligante asfáltico e, por consequência, o desequilíbrio entre os fenômenos de volatilização e de oxidação.

Os ensaios MSCR (ASTM D7405, 2010) foram conduzidos em um reômetro de cisalhamento dinâmico da empresa TA Instruments, modelo AR-2000ex, em cinco temperaturas igualmente espaçadas de $6^{\circ} \mathrm{C}(52,58,64,70$ e $76^{\circ} \mathrm{C}$ ). Apenas condições padronizadas de ensaio foram consideradas no estudo, ou seja, tempo de fluência de $1 \mathrm{~s}$, tempo de recuperação de 9s, níveis de tensão de 100 e $3.200 \mathrm{~Pa}, 10$ ciclos de fluência e de recuperação para cada nível e materiais envelhecidos a curto prazo. Duas réplicas foram feitas para cada ligante asfáltico e os resultados finais do percentual de recuperação $\mathrm{R}$ e da compliância não-recuperável $\mathrm{J}_{\mathrm{nr}}$ correspondem à média aritmética simples destas réplicas. As diferenças percentuais entre as compliâncias $\left(\mathrm{J}_{\mathrm{nr}, \text { diff }}\right)$, que expressam o aumento percentual de $\mathrm{J}_{\mathrm{nr}}$ após o aumento do nível de tensão de 100 para 3.200Pa, foram calculadas conforme a Equação 1 para todas as temperaturas. Os resultados foram comparados com o limite máximo de $75 \%$, estipulado pela norma AASHTO MP19 (2010).

$$
\begin{aligned}
& \left.\mathrm{J}_{\mathrm{nr}, \text { diff }}=\left[\left(\mathrm{J}_{\mathrm{nr}} 3200-\mathrm{J}_{\mathrm{nr}} 100\right) / \mathrm{J}_{\mathrm{nr}} 100\right)\right] .100 \\
& \text { em que: }
\end{aligned}
$$

$J_{n r}$, diff: diferença percentual entre as compliâncias, em porcentagem;

$J_{n r} 3200$ : compliância não-recuperável a 3.200

$\mathrm{Pa}\left[\mathrm{Pa}^{-1}\right.$ ou kPa $\left.{ }^{-1}\right]$;

$J_{n r} 100$ : compliância não-recuperável a 100

$\mathrm{Pa}\left[\mathrm{Pa}^{-1}\right.$ ou $\left.\mathrm{kPa}^{-1}\right]$.

Da mesma maneira, as diferenças percentuais entre as recuperações dos ligantes asfálticos a 100 e a $3.200 \mathrm{~Pa}$ $\left(\mathrm{R}_{\text {diff }}\right)$ foram determinadas conforme a Equação 2 com o propósito de verificar o impacto do aumento do nível de tensão nos valores de $\mathrm{R}$ dos materiais.

$$
\mathrm{R}_{\mathrm{diff}}=[(\mathrm{R} 100-\mathrm{R} 3200) / \mathrm{R} 100] .100
$$
em que:

$R_{\text {diff: }}$ diferença percentual entre as recuperações, em porcentagem;

R100: percentual de recuperação a $100 \mathrm{~Pa}$, em \%;

R3200: percentual de recuperação a 3.200Pa, em \%.

Além das alterações nos critérios de avaliação da suscetibilidade à deformação permanente e da estrutura/extensão da rede polimérica nos ligantes asfálticos modificados, a incorporação do MSCR à especificação Superpave $^{\circledR}$ também levou à atribuição de níveis de tráfego adequados aos materiais em função do valor de $\mathrm{J}_{\mathrm{nr}}$ a 3.200 $\mathrm{Pa}$ e na temperatura alta do $\mathrm{PG}$ do pavimento. Estes níveis, designados pelas letras $\mathrm{S}$ (padrão), $\mathrm{H}$ (pesado), $\mathrm{V}$ (muito pesado) e E (extremamente pesado), são associados a números de passadas de eixo-padrão simples (ESALs equivalent single-axle loads) e/ou a velocidades dos veículos na via (Gierhart, 2013; Bahia, 2014), conforme Tabela 2. Este procedimento substituiu uma prática anteriormente adotada nos Estados Unidos para lidar com tráfegos mais pesados e/ou velocidades mais baixas, conhecida como grade-bumping e segundo a qual o aumento de um grau PG na classificação do CAP dobraria a sua rigidez e o tornaria apto a suportar carregamentos mais severos no pavimento (D'Angelo, 2010).

\section{APRESENTAÇÃO E DISCUSSÃO DOS RESULTADOS}

\subsection{Ensaios tradicionais e de viscosidade rotacional}

A Tabela 3 destaca os resultados dos ensaios de penetração a $25^{\circ} \mathrm{C}$, de ponto de amolecimento e de viscosidade rotacional a $135^{\circ} \mathrm{C}$ para todos os ligantes asfálticos, bem como os respectivos parâmetros de sensibilidade ao 
Tabela 2. Níveis e velocidades de tráfego adequados ao CAP em função do valor de $\mathrm{J}_{\mathrm{nr}}$ a 3.200Pa e na temperatura do PG (Gierhart, 2013; Bahia, 2014)

\begin{tabular}{lcl}
\hline & $J_{\text {nr }}$ máximo a & \\
nível de tráfego & $\left.\mathbf{3 . 2 0 0 P a}(\boldsymbol{k P a})^{-1}\right)$ & número de eixos-padrão simples (ESALs) e/ou velocidade do tráfego \\
\hline padrão $(\mathrm{S})$ & 4,0 & inferior a 10 milhões e tráfego em velocidade padrão (média superior a 70km/h) \\
pesado $(\mathrm{H})$ & 2,0 & entre 10 e 30 milhões ou tráfego em velocidade baixa (média entre 20 e $70 \mathrm{~km} / \mathrm{h})$ \\
muito pesado $(\mathrm{V})$ & 1,0 & superior a 30 milhões ou tráfego em velocidade muito baixa $($ média inferior a $20 \mathrm{~km} / \mathrm{h})$ \\
extremamente pesado $(\mathrm{E})$ & 0,5 & superior a 30 milhões e tráfego em velocidade muito baixa (média inferior a 20km/h) \\
\hline
\end{tabular}

Tabela 3. Resultados dos ensaios tradicionais e de viscosidade rotacional e parâmetros de sensibilidade ao envelhecimento a curto prazo

\begin{tabular}{|c|c|c|c|c|}
\hline propriedade reológica ou parâmetro & $50 / 70$ & $C A P+P P A$ & $C A P+S B R$ & $C A P+S B R+P P A$ \\
\hline penetração @ $25^{\circ} \mathrm{C}$ virgem $(\mathrm{dmm})$ & 58,0 & 36,5 & 39,0 & 47,0 \\
\hline penetração @ $25^{\circ} \mathrm{C}$ RTFOT (dmm) & 30,8 & 23,8 & 37,0 & 31,3 \\
\hline ponto de amolecimento virgem (PA, $\left.{ }^{\circ} \mathrm{C}\right)$ & 49,4 & 56,8 & 63,3 & 57,3 \\
\hline ponto de amolecimento RTFOT $\left(\mathrm{PA},{ }^{\circ} \mathrm{C}\right)$ & 56,1 & 67,2 & 65,7 & 66,0 \\
\hline viscosidade @ $135^{\circ} \mathrm{C}$ virgem (Pa.s) & 0,36 & 0,72 & 1,13 & 1,11 \\
\hline viscosidade @ $135^{\circ} \mathrm{C}$ RTFOT (Pa.s) & 0,59 & 1,94 & 1,83 & 3,16 \\
\hline penetração retida $\mathrm{R}_{\mathrm{PEN}}(\%)$ & 53,0 & 65,1 & 94,9 & 66,5 \\
\hline aumento do PA $\left(\mathrm{I}_{\mathrm{R} \& \mathrm{~B}},{ }^{\circ} \mathrm{C}\right)$ & 6,8 & 10,4 & 2,5 & 8,7 \\
\hline índice de aumento da viscosidade $\left(\mathrm{R}_{\mathrm{V}}\right)^{*}$ & 1,63 & 2,68 & 1,61 & 2,85 \\
\hline
\end{tabular}

* Razão entre as viscosidades rotacionais após e antes do envelhecimento a curto prazo.

envelhecimento a curto prazo. Como esperado, a adição de um ou dois modificadores ao CAP de base provocou redução na penetração e aumento no ponto de amolecimento e na viscosidade rotacional, o que indica um enrijecimento da formulação. Estes efeitos são potencializados pelo envelhecimento a curto prazo, especialmente no caso do ponto de amolecimento e da viscosidade rotacional. Em linhas gerais, pode-se dizer que o nível de enrijecimento é maior após a adição de PPA ao CAP porque o CAP+PPA apresenta os menores valores de penetração em ambas as condições de envelhecimento $(36,5 \mathrm{dmm}$ na condição virgem e $23,8 \mathrm{dmm}$ na envelhecida a curto prazo) e o maior ponto de amolecimento na condição envelhecida $\left(67,2^{\circ} \mathrm{C}\right)$.

Uma análise mais criteriosa dos resultados apresentados na Tabela 3 mostra que o nível de enrijecimento após a adição de modificadores é maior para o CAP+SBR do que para o $\mathrm{CAP}+\mathrm{SBR}+\mathrm{PPA}$ antes do envelhecimento a curto prazo, o contrário sendo observado após o envelhecimento. Isto indica que a presença do PPA está, de alguma forma, relacionada ao aumento mais expressivo da rigidez do CAP quando o material é submetido ao envelhecimento acelerado em laboratório. Tal observação ganha ainda mais relevância quando se observam as variações relativamente significativas das propriedades reológicas do CAP+PPA após o envelhecimento: aumento do ponto de amolecimento em mais de $10^{\circ} \mathrm{C}\left(10,4^{\circ} \mathrm{C}\right)$, aumento da viscosidade rotacional em mais de $100 \%$ (valor de $\mathrm{R}_{\mathrm{V}}$ superior a 2,0) e redução da penetração em mais de $30 \%$ (valor de $\mathrm{R}_{\mathrm{PEN}}$ inferior a $70 \%$ ). Em termos científicos, o processo de reação química entre o PPA e o CAP ainda não é compreendido integralmente pelos pesquisadores; por isso, diversas teorias têm sido postuladas na literatura. Uma delas afirma que a presença do PPA leva a uma separação dos conglomerados de asfaltenos encontrados no material de base e, uma vez separados, estas moléculas individuais se dispersam na fase maltênica e aumentam o seu volume efetivo no CAP (Lesueur, 2009; Fee et al., 2010). Este aumento significativo do volume das moléculas de asfaltenos no CAP indica um envelhecimento precoce do material modificado, o que pode levar à ruptura do pavimento antes do final da vida útil de serviço (Tam, 2012).

Os parâmetros de avaliação da sensibilidade dos ligantes asfálticos ao envelhecimento a curto prazo mostram que esta sensibilidade é menor para o CAP+SBR. Chama a atenção o aumento significativo desta sensibilidade quando o teor de SBR é reduzido e o PPA é adicionado ao $\mathrm{CAP}+\mathrm{SBR}$ para produzir o $\mathrm{CAP}+\mathrm{SBR}+\mathrm{PPA}$ : o parâmetro $\mathrm{R}_{\mathrm{PEN}}$ é reduzido em aproximadamente $30 \%$ (de 94,9 para $66,5 \%$ ), o valor de $I_{R \& B}$ aumenta em mais de $6^{\circ} \mathrm{C}$ (de 2,5 para $8,7^{\circ} \mathrm{C}$ ) e o valor de $\mathrm{R}_{\mathrm{V}}$ aumenta em mais de $70 \%$ (de 1,61 para 2,85). Uma vez que os teores de SBR e de PPA variam simultaneamente do CAP+SBR $(5,5 \%$ de SBR) para o $\mathrm{CAP}+\mathrm{SBR}+\mathrm{PPA}(3,5 \%$ de SBR e $0,5 \%$ de PPA), não é possível afirmar que o PPA é o único responsável por este aumento da sensibilidade do CAP+SBR ao envelhecimento, embora o impacto da sua adição na sensibilidade do CAP puro seja reconhecido como expressivo em algumas propriedades reológicas.

\subsection{Ensaios de fluência e de recuperação sob tensão múltipla (MSCR)}

\subsubsection{Percentuais de recuperação}

A Figura 1 apresenta os percentuais de recuperação do CAP puro, do CAP+PPA, do CAP+SBR e do CAP+SBR+PPA. Em um contexto geral, a adição dos modificadores ao CAP de base proporciona um aumento dos valores de $\mathrm{R}$ do material, especialmente nas temperaturas de até $70^{\circ} \mathrm{C}$. Percentuais de recuperação maiores podem ser traduzidos como uma parcela maior de deformação recuperável em relação ao total sofrido pelo material após cada ciclo de fluência e de recuperação (o acúmulo de deformação plástica é menor), o que é favorável à resistência à deformação permanente. À exceção da temperatura de $52^{\circ} \mathrm{C}$, estes percentuais são maiores para $\mathrm{o}$ $\mathrm{CAP}+\mathrm{SBR}+\mathrm{PPA}$ se comparado ao CAP+PPA, tanto a 100 quanto a $3.200 \mathrm{~Pa}$. Os menores percentuais (até $44 \%$ a $100 \mathrm{~Pa}$ e até $40 \%$ a $3.200 \mathrm{~Pa}$ ) são observados para o $\mathrm{CAP}+\mathrm{SBR}$ em todas as temperaturas e níveis de tensão dentre os ligantes asfálticos modificados, sendo este o material que recupera menos nestas condições. No caso do CAP puro, os resultados não superam 13\% a 100Pa e $9 \%$ a 3.200Pa.

Apesar das diferenças entre os teores de SBR e de PPA no $\mathrm{CAP}+\mathrm{SBR}+\mathrm{PPA}(3,5 \%$ de SBR e $0,5 \%$ de PPA) e no CAP+PPA (1,2\% de PPA), os valores de $\mathrm{R}$ são muito próximos entre si para os dois materiais nas temperaturas 


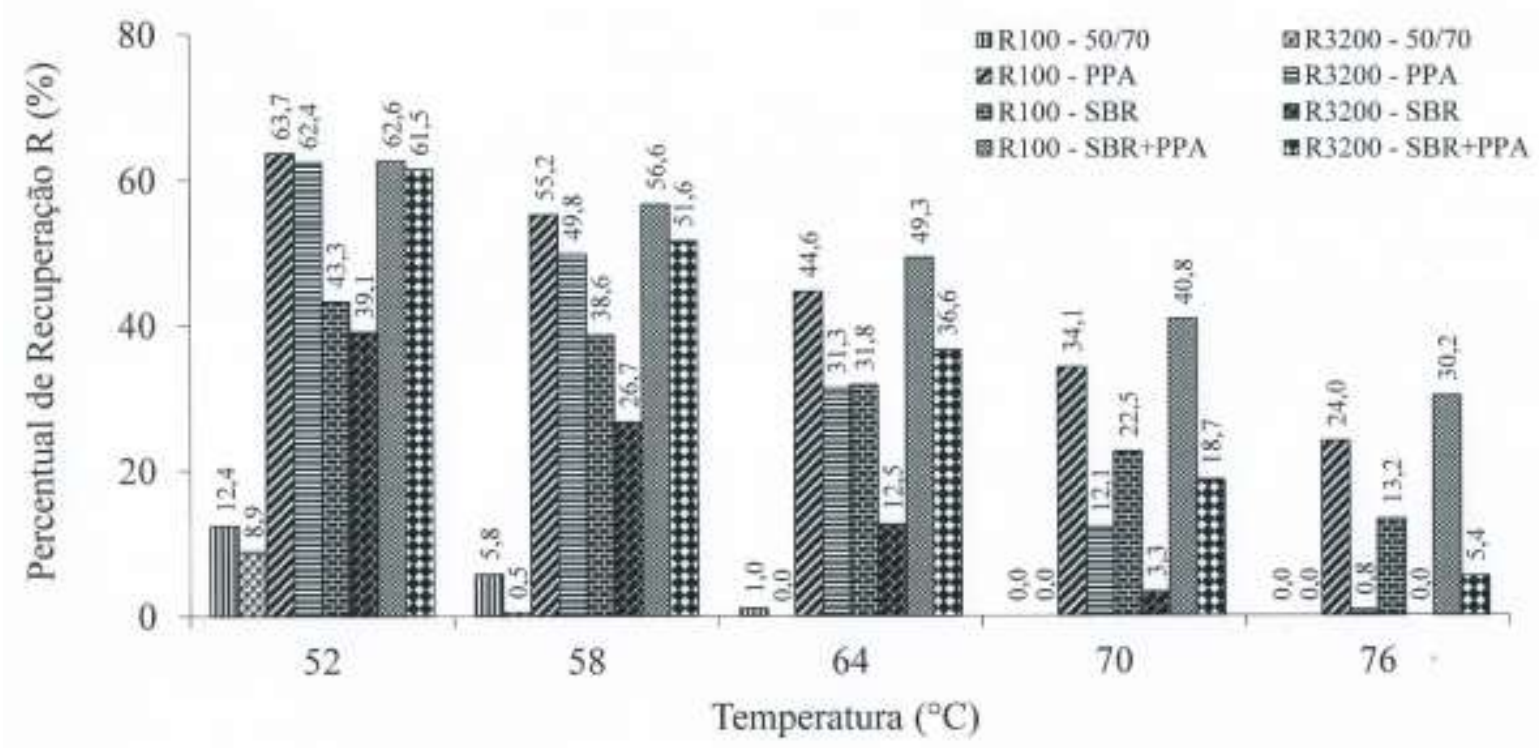

Figura 1. Percentuais de recuperação a $100 \mathrm{~Pa}(\mathrm{R} 100)$ e a $3.200 \mathrm{~Pa}(\mathrm{R} 3200)$ do CAP puro 50/70 e das formulações com PPA, SBR e SBR+PPA

de 52 e $58^{\circ} \mathrm{C}$ a 100 e a $3.200 \mathrm{~Pa}$, de modo que ambas as formulações podem ser consideradas equivalentes à luz destes resultados. Os decréscimos com a temperatura se mostram parecidos para os três ligantes asfálticos modificados nas temperaturas de 64,70 e $76^{\circ} \mathrm{C}$ e a $100 \mathrm{~Pa}$, o mesmo sendo observado para o CAP+PPA e para o CAP+SBR+PPA a 3.200Pa. Já para o material puro, estes percentuais são não-nulos apenas nas temperaturas mais baixas do MSCR (até $64^{\circ} \mathrm{C}$ a $100 \mathrm{~Pa}$ e até $58^{\circ} \mathrm{C}$ a $3.200 \mathrm{~Pa}$ ), sendo iguais a zero em todas as demais condições de temperatura e de tensão. As diferenças entre os valores de $\mathrm{R}$ a 100 e a $3.200 \mathrm{~Pa}$ são relativamente pequenas para um mesmo ligante asfáltico nas temperaturas de até $58^{\circ} \mathrm{C}$ (exceção feita ao CAP+SBR), passando a ser significativas nas outras temperaturas de ensaio.

Uma constatação interessante sobre os percentuais de recuperação do ligante asfáltico puro e dos modificados com PPA, SBR e SBR+PPA pode ser visualizada quando estes materiais são ensaiados a $76^{\circ} \mathrm{C}$ e a $3.200 \mathrm{~Pa}$. Embora preparadas com modificadores de naturezas e teores distintos, os percentuais são todos nulos ou muito pequenos (até $6 \%$ ) para as três formulações e o CAP de base nas condições de ensaio estabelecidas pela especificação Superpa$\mathrm{ve}^{\circledR}$. Isto significa que a adição dos modificadores nos teores preestabelecidos foi suficiente para aumentar a temperatura do PG do CAP de 64 para 76, porém não se mostrou suficiente para melhorar a resposta elástica do material em condições muito críticas de tensão e de temperatura. Outra possível explicação está relacionada às propriedades intrínsecas do CAP e dos modificadores, de modo que, para tais situações, a escolha de outros tipos de CAP e/ou de modificadores seria capaz de fornecer à formulação níveis mais elevados de resposta elástica. Uma possível solução para este caso seria o aumento das quantidades de SBR e/ou de PPA no CAP+PPA, no CAP+SBR e no $\mathrm{CAP}+\mathrm{SBR}+\mathrm{PPA}$; entretanto, a classificação PG 76$\mathrm{xx}$ poderia não se manter a mesma para os três ligantes asfálticos modificados, especialmente no caso do $\mathrm{CAP}+\mathrm{SBR}+\mathrm{PPA}$ (grau PG contínuo muito próximo de $\left.82^{\circ} \mathrm{C}\right)$.

\subsubsection{Compliâncias não-recuperáveis}

A Figura 2 mostra os valores das compliâncias nãorecuperáveis para o $\mathrm{CAP}$ de base, o CAP+PPA, o $\mathrm{CAP}+\mathrm{SBR}$ e o $\mathrm{CAP}+\mathrm{SBR}+\mathrm{PPA}$. O efeito da adição dos modificadores nas compliâncias não-recuperáveis do CAP é o contrário do observado no percentual de recuperação, ou seja, a presença do (s) modificador (es) causa uma redução desta compliância em todas as temperaturas e níveis de tensão. Valores mais baixos de $\mathrm{J}_{\mathrm{nr}}$ podem ser traduzidos como uma suscetibilidade menor do ligante asfáltico à deformação permanente em campo ou, em outras palavras, há um aumento da contribuição do CAP na resistência da mistura asfáltica à deformação permanente após a adição de modificadores. Os resultados oscilam entre 0,03 e $2,38 \mathrm{kPa}^{-1}$ para o CAP+PPA e para o $\mathrm{CAP}+\mathrm{SBR}+\mathrm{PPA}$ e podem superar os $3,00 \mathrm{kPa}^{-1}$ para $\mathrm{o}$ $\mathrm{CAP}+\mathrm{SBR}$ em temperaturas mais elevadas. No caso do CAP sem modificação, estas compliâncias superam os $5,00 \mathrm{kPa}^{-1}$ nas temperaturas de 70 e de $76^{\circ} \mathrm{C}$ a $100 \mathrm{~Pa}$ e podem atingir os $15,00 \mathrm{kPa}^{-1}$ nas temperaturas acima de $70^{\circ} \mathrm{C}$ e a $3.200 \mathrm{~Pa}$.

Ainda sobre os valores das compliâncias nãorecuperáveis, é possível observar que os resultados são relativamente próximos entre si para o $\mathrm{CAP}+\mathrm{PPA}$, o $\mathrm{CAP}+\mathrm{SBR}$ e o $\mathrm{CAP}+\mathrm{SBR}+\mathrm{PPA}$ nas temperaturas de até $64^{\circ} \mathrm{C}$ a $100 \mathrm{~Pa}$ e de até $58^{\circ} \mathrm{C}$ a $3.200 \mathrm{~Pa}$. Em outras palavras, a distinção entre os efeitos das modificações não é tão simples de ser feita quando a temperatura do ensaio MSCR é mais baixa. A proximidade dos resultados se mantém ao longo de todo o espectro de temperaturas para as duas formulações com PPA (CAP+PPA e $\mathrm{CAP}+\mathrm{SBR}+\mathrm{PPA}$ ), tanto a 100 quanto a 3.200Pa. Estes resultados parecidos de $\mathrm{J}_{\mathrm{nr}}$ em temperaturas mais baixas podem ser atribuídos aos níveis muito baixos de deformação observados nos ligantes asfálticos modificados, o que impede uma visualização clara das respostas normalizadas de cada formulação. Esta distinção passa a ser mais visível à medida que a temperatura de ensaio - e, por consequência, o nível de deformação - aumenta, ou seja, as respostas normalizadas passam a refletir com mais clareza o (s) tipo 


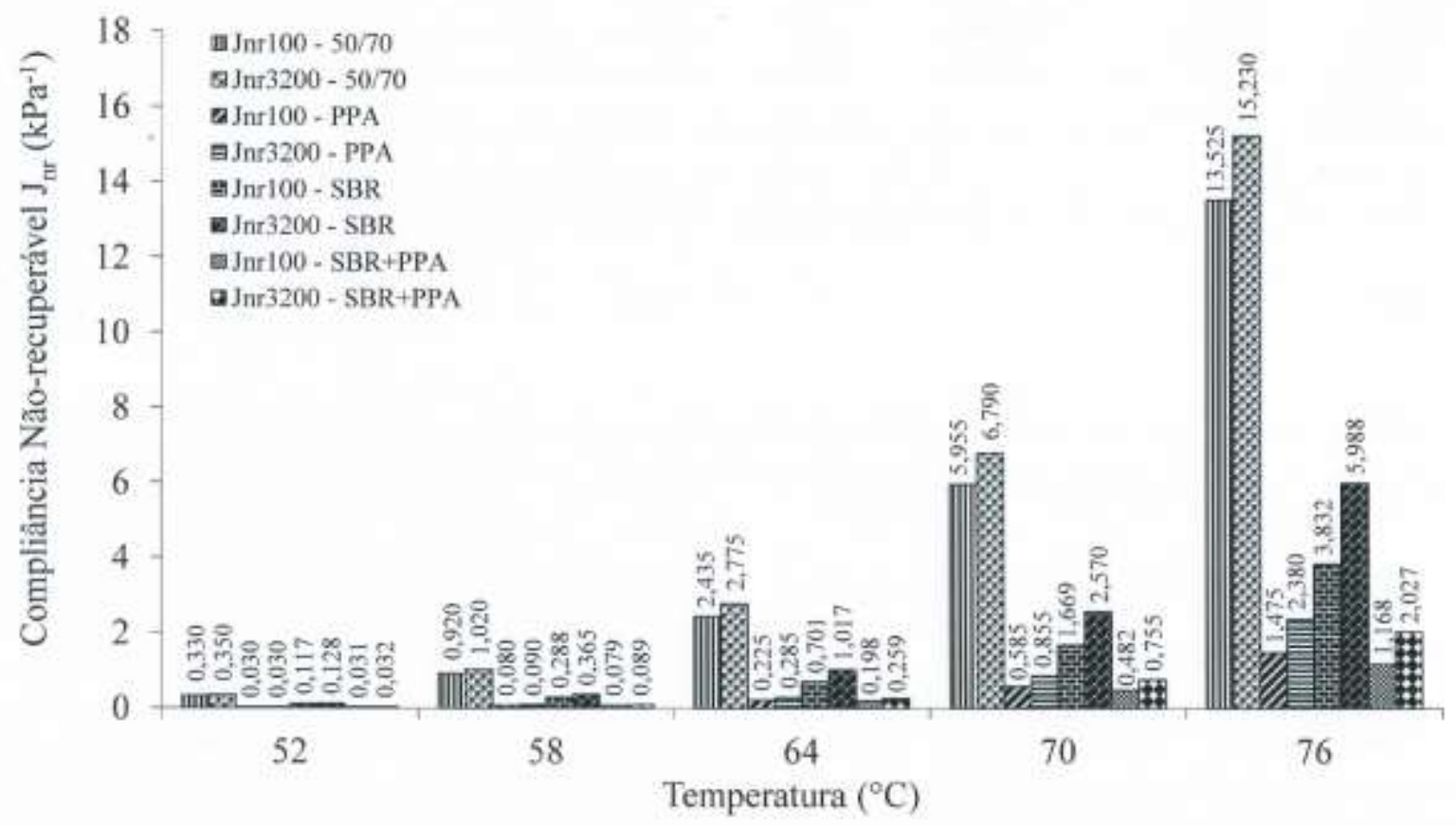

Figura 2. Compliâncias não-recuperáveis a 100Pa $\left(\mathrm{J}_{n r} 100\right)$ e a 3.200Pa $\left(\mathrm{J}_{n r} 3200\right)$ do CAP puro 50/70 e das formulações com PPA, SBR e SBR+PPA

(s) de modificador (es) adicionados ao CAP quando a temperatura de ensaio é maior.

Em uma comparação direta dos resultados de $\mathrm{J}_{\mathrm{nr}}$ para as duas formulações com SBR, observa-se que a adição de PPA e a redução do teor de copolímero foram benéficas para a formulação porque houve reduções significativas desta propriedade em temperaturas típicas de ocorrência da deformação permanente no pavimento. Não se pode, entretanto, afirmar que apenas o aumento do teor de PPA ou a redução no teor de SBR - contribuiu para esta redução de $\mathrm{J}_{\mathrm{nr}}$, pois ambos os teores variaram de uma formulação para outra. Uma hipótese plausível é a de que o PPA possui uma parcela de contribuição nesta redução da suscetibilidade do CAP à deformação permanente, especialmente quando são analisados os efeitos da sua adição nas compliâncias não-recuperáveis do material de base (reduções superiores a 80\%). Como destacado anteriormente, os aumentos da rigidez e da consistência do ligante asfáltico após a adição do PPA podem ser explicados pela quebra dos conglomerados de asfaltenos e pelo aumento do volume efetivo destas moléculas na fase maltênica do material de base (Lesueur, 2009; Fee et al., 2010). A estrutura coloidal do CAP também permite que o polímero e o PPA atuem de maneira cooperada e mais eficiente na modificação das propriedades originais do material, considerando a hipótese de que os efeitos das modificações individuais são somados e resultam nas propriedades finais da formulação (Lesueur, 2009).

\subsubsection{Diferenças percentuais}

Os resultados das diferenças percentuais entre as compliâncias não-recuperáveis ( $\left.\mathrm{J}_{\mathrm{nr} \text {, diff }}\right)$ e entre os percentuais de recuperação $\left(\mathrm{R}_{\text {diff }}\right)$ do CAP puro, do CAP+PPA, do $\mathrm{CAP}+\mathrm{SBR}$ e do $\mathrm{CAP}+\mathrm{SBR}+\mathrm{PPA}$ são apresentados na Tabela 4. Alguns valores de $\mathrm{R}_{\text {diff }}$ não puderam ser calculados - ou não foram inseridos na tabela - porque o percentual de recuperação do ligante asfáltico a 3.200Pa é nulo. Os maiores valores de $J_{n r}$, diff são observados para o
$\mathrm{CAP}+\mathrm{SBR}$ nas temperaturas de até $64^{\circ} \mathrm{C}$, ao passo que o $\mathrm{CAP}+\mathrm{SBR}+\mathrm{PPA}$ apresenta estes maiores valores a 70 e a $76^{\circ} \mathrm{C}$. O CAP puro apresenta os menores valores em um contexto geral, seguido pelo CAP+PPA e pelas duas formulações com SBR. Embora maiores do que os resultados encontrados para o CAP puro, nenhum dos ligantes asfálticos modificados apresenta uma sensibilidade excessiva à tensão $\left(\mathrm{J}_{\mathrm{nr} \text {, diff }}>75 \%\right.$ na temperatura do $\mathrm{PG}$ e a $\left.3.200 \mathrm{~Pa}\right)$ segundo os critérios da especificação Superpave ${ }^{\circledR}$. O resultado obtido para o CAP+SBR+PPA é o que mais se aproxima deste limite $(73,6 \%)$, seguido pelo CAP+PPA $(61,4 \%)$, pelo CAP+SBR $(56,2 \%)$ e finalmente pelo CAP puro $(14,0 \%)$.

Com relação aos resultados de $\mathrm{R}_{\text {diff, observa-se uma }}$ redução deste valor a 52 e a $58^{\circ} \mathrm{C}$ após a adição dos modificadores ao CAP, especialmente no caso do $\mathrm{CAP}+\mathrm{SBR}+\mathrm{PPA}$ e do $\mathrm{CAP}+\mathrm{PPA}$. Isto significa que, do ponto de vista do percentual de recuperação, o processo de modificação do ligante asfáltico levou a uma redução da sensibilidade ao aumento do nível de tensão de 100 para 3.200Pa. Estes percentuais oscilam entre 2 e $97 \%$ para o CAP+PPA, entre 9 e $86 \%$ para o CAP+SBR e entre 1 e $83 \%$ para o CAP+SBR+PPA, sendo menores para a formulação com SBR+PPA e maiores para a formulação somente com SBR. Interessante observar que os efeitos das modificações nos dois parâmetros reológicos do ligante asfáltico $\left(\mathrm{R}\right.$ e $\mathrm{J}_{\mathrm{nr}}$ ) são distintos, ou seja, a modificação aumentou a sensibilidade do CAP à tensão do ponto de vista da compliância não-recuperável ( $J_{n r}$, diff maior) e a reduziu do ponto de vista do percentual de recuperação $\left(\mathrm{R}_{\text {diff }}\right.$ menor). Entretanto, para fins de especificação Superpave ${ }^{\circledR}$, apenas o valor de $J_{n r}$ diff na temperatura do PG e a 3.200Pa é levado em consideração.

A adição de PPA e a redução do teor de copolímero SBR na formulação CAP+SBR, do ponto de vista de sensibilidade à temperatura, efeitos mistos nos resultados

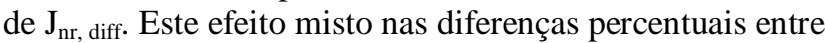
as compliâncias não-recuperáveis pode ser traduzido em 
DOMINGOS, M. D. I.; FAXINA, A. L.

Tabela 4. Valores de $\mathrm{R}_{\text {diff }}$ e de $\mathrm{J}_{\mathrm{nr} \text { diff }}$ para os ligantes asfálticos envelhecidos a curto prazo

\begin{tabular}{|c|c|c|c|c|c|}
\hline parâmetro & temperatura $\left({ }^{\circ} \mathrm{C}\right)$ & $50 / 70$ & $C A P+P P A$ & $\mathrm{CAP}+\mathrm{SBR}$ & $C A P+S B R+P P A$ \\
\hline \multirow{4}{*}{$\begin{array}{l}\text { diferença percentual entre as recupera- } \\
\text { ções a } 100 \text { e a } 3.200 \mathrm{~Pa}\left(\mathrm{R}_{\text {diff }}\right) \text {, em \% }\end{array}$} & 52 & 28,4 & 2,0 & 9,8 & 1,9 \\
\hline & 58 & 91,5 & 9,8 & 31,0 & 8,9 \\
\hline & 70 & - & 64,5 & 85,6 & 54,2 \\
\hline & 76 & - & 96,8 & - & 82,2 \\
\hline \multirow{4}{*}{$\begin{array}{l}\text { diferença percentual entre as compliân- } \\
\text { cias não-recuperáveis a } 100 \text { e a } 3.200 \\
\mathrm{~Pa}\left(\mathrm{~J}_{\mathrm{nr} \text {, diff }}\right) \text {, em \% }\end{array}$} & 52 & 6,1 & 0,0 & 9,5 & 3,4 \\
\hline & 64 & 14,0 & 26,7 & 45,1 & 30,9 \\
\hline & 70 & 14,0 & 46,2 & 53,9 & 56,6 \\
\hline & 76 & 12,6 & 61,4 & 56,2 & 73,6 \\
\hline
\end{tabular}

valores maiores a 70 e a $76^{\circ} \mathrm{C}$, valores numéricos menores nas demais temperaturas e uma variação maior dos resultados com o incremento da temperatura. Uma vez que o parâmetro $\mathrm{J}_{\mathrm{nr} \text {, diff }}$ avalia a resposta não-linear do CAP e o nível de rearranjo das moléculas de polímero após o incremento da tensão (Anderson, 2011) e os percentuais de recuperação do $\mathrm{CAP}+\mathrm{SBR}+\mathrm{PPA}$ são maiores do que os obtidos para o CAP+SBR (Figura 1), pode-se inferir que a presença do PPA contribuiu - pelo menos parcialmente para uma melhor compatibilidade do modificador com o CAP e a formação de uma rede polimérica mais extensa na formulação, o que está em concordância com as análises presentes na publicação de Zhang e Yu (2010).

\subsubsection{Níveis adequados de tráfego para os ligantes asfálticos}

A Tabela 5 mostra os níveis de tráfego recomendados para os ligantes asfálticos em todas as temperaturas de realização do MSCR, considerando as categorias e os limites máximos e mínimos de $\mathrm{J}_{\mathrm{nr}}$ a 3.200Pa discriminados na Tabela 2. Cabe destacar que, diferentemente do procedimento estabelecido na especificação Superpave ${ }^{\circledR}$, admitiu-se neste presente trabalho que os critérios para atribuição do tráfego podem ser estendidos às demais temperaturas do MSCR que não apenas a temperatura alta do PG do pavimento. $\mathrm{O}$ uso do $\mathrm{CAP}$ de base não é recomendado nem mesmo em condições-padrão de tráfego a 70 e a $76^{\circ} \mathrm{C}$, o mesmo ocorrendo com o $\mathrm{CAP}+\mathrm{SBR}$ a $76^{\circ} \mathrm{C}$. Por outro lado, os níveis de tráfego são significativamente maiores (e iguais) para o CAP+PPA e para o $\mathrm{CAP}+\mathrm{SBR}+\mathrm{PPA}$ nas temperaturas mais altas do ensaio MSCR: extremamente pesado a $64^{\circ} \mathrm{C}$, muito pesado a $70^{\circ} \mathrm{C}$ e padrão a $76^{\circ} \mathrm{C}$.

Duas observações podem ser destacadas com base nos resultados de tráfego: (a) o uso do PPA como uma alternativa à modificação do ligante asfáltico com polímeros se mostra interessante do ponto de vista de resistência a carregamentos mais lentos e/ou número maior de passadas de um eixo-padrão simples; e (b) a adição do PPA e a redução do teor de SBR foram benéficas ao CAP+SBR porque os tráfegos sofreram aumentos substanciais, sobre- tudo a 64 e a $70^{\circ} \mathrm{C}$ (dois níveis em cada temperatura).

\section{CONCLUSÕES}

Com base nos resultados obtidos neste trabalho, as seguintes conclusões podem ser apontadas no que diz respeito aos comportamentos fluência-recuperação de ligantes asfálticos modificados com SBR, PPA e SBR+PPA:

- a adição dos modificadores trouxe benefícios ao ligante asfáltico de base 50/70 do ponto de vista de resistência à deformação permanente, tendo em vista que os percentuais de recuperação (R) aumentaram e as compliâncias não-recuperáveis $\left(\mathrm{J}_{\mathrm{nr}}\right)$ diminuíram em temperaturas variando entre 52 e $76^{\circ} \mathrm{C}$; os resultados foram melhores para 0 $\mathrm{CAP}+\mathrm{SBR}+\mathrm{PPA}$ e para o $\mathrm{CAP}+\mathrm{PPA}$ se comparados àqueles obtidos para o $\mathrm{CAP}+\mathrm{SBR}$, sendo que a formulação com SBR+PPA apresenta valores maiores de $\mathrm{R}$ e menores de $\mathrm{J}_{\mathrm{nr}}$ em comparação ao material modificado somente com PPA;

- os valores de R são nulos ou muito pequenos para as três formulações a $76^{\circ} \mathrm{C}$ e a $3.200 \mathrm{~Pa}$, o que pode ser atribuído às características intrínsecas dos modificadores ou aos teores de cada aditivo (suficientes para elevar o PG do CAP de 64 para 76, porém insuficientes para possibilitar ganhos significativos para o parâmetro $\mathrm{R}$ nas condições mais críticas de ensaio); os valores de $\mathrm{J}_{\mathrm{nr}}$ são muito parecidos para o $\mathrm{CAP}+\mathrm{PPA}$, o $\mathrm{CAP}+\mathrm{SBR}$ e o $\mathrm{CAP}+\mathrm{SBR}+\mathrm{PPA}$ nas temperaturas iguais ou inferiores a $64^{\circ} \mathrm{C}$, o que pode ser explicado pelos níveis muito baixos de deformação observados nos ligantes asfálticos modificados durante o ensaio e pela consequente dificuldade em distinguir uma formulação da outra;

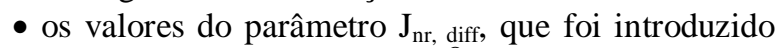
na especificação Superpave ${ }^{\circledR}$ para avaliar a nãolinearidade da resposta do ligante asfáltico e o nível de rearranjo das cadeias poliméricas da formulação após o aumento do nível de tensão, são todos inferiores a $75 \%$ para os materiais estudados e, segundo

Tabela 5. Níveis de tráfego recomendados para os ligantes asfálticos em função do valor da compliância não-recuperável $\left(\mathrm{J}_{\mathrm{nr}}\right)$ a $3.200 \mathrm{~Pa}$

\begin{tabular}{ccccc}
\hline \multirow{2}{*}{ temperatura $\left({ }^{\circ} \boldsymbol{C}\right)$} & \multicolumn{5}{c}{ nível de tráfego $(\boldsymbol{S}, \boldsymbol{H}, \boldsymbol{V} \text { ou } \mathrm{E})^{*}$} \\
\cline { 2 - 5 } & $\boldsymbol{C A P} \mathbf{5 0 / 7 0}$ & $\boldsymbol{C A P + P P A}$ & $\boldsymbol{C A P + S B R}$ & $\boldsymbol{C A P + S B R + P P A}$ \\
\hline 52 & $\mathrm{E}$ & $\mathrm{E}$ & $\mathrm{E}$ & $\mathrm{E}$ \\
58 & $\mathrm{H}$ & $\mathrm{E}$ & $\mathrm{E}$ & $\mathrm{E}$ \\
64 & $\mathrm{~S}$ & $\mathrm{E}$ & $\mathrm{H}$ & $\mathrm{E}$ \\
70 & - & $\mathrm{V}$ & $\mathrm{S}$ & $\mathrm{V}$ \\
76 & - & $\mathrm{S}$ & - & $\mathrm{S}$
\end{tabular}

* $\mathrm{S}=$ tráfego padrão; $\mathrm{H}=$ tráfego pesado; $\mathrm{V}=$ tráfego muito pesado; $\mathrm{E}=$ tráfego extremamente pesado 
os critérios Superpave ${ }^{\circledR}$, estes materiais têm uma condição aceitável de sensibilidade à tensão e podem ser empregados em pavimentação; em um contexto geral, os parâmetros reológicos do $\mathrm{CAP}+\mathrm{SBR}+\mathrm{PPA}$ e do CAP+PPA (R e $\mathrm{J}_{\mathrm{nr}}$ ) são menos sensíveis ao aumento da tensão se comparados àqueles obtidos para o $\mathrm{CAP}+\mathrm{SBR}$, o que indica que a adição do PPA e a redução do teor de SBR reduziram a sensibilidade da formulação ao incremento da tensão; e

- a verificação dos níveis de tráfego adequados aos ligantes asfálticos sugere que o CAP+PPA e o $\mathrm{CAP}+\mathrm{SBR}+\mathrm{PPA}$ são capazes de suportar os mesmos níveis em todo o espectro de temperaturas de 52 a $76^{\circ} \mathrm{C}$, e as diferenças entre os tráfegos recomendados para ambos os materiais e para o $\mathrm{CAP}+\mathrm{SBR}$ são de dois níveis nas temperaturas de 64 e $70^{\circ} \mathrm{C}$ (de muito pesado para extremamente pesado) e de "sem classificação de tráfego" para tráfego padrão a $76^{\circ} \mathrm{C}$; em outras palavras, a presença do PPA e o menor teor de SBR contribuíram positivamente para o aumento da capacidade de resistência do CAP+SBR ao tráfego (maior número de passadas de eixo-padrão simples e/ou menor velocidade média dos veículos). Os resultados semelhantes das duas formulações com PPA sugerem que o ácido tem potencial para substituir o uso de SBR na modificação de ligantes asfálticos, desde que a formulação não apresente resistências muito baixas aos outros mecanismos de ruptura do pavimento (trincas por fadiga e trincas de origem térmica).

\section{AGRADECIMENTOS}

O primeiro autor agradece à Fundação de Amparo à Pesquisa do Estado de São Paulo (FAPESP) pela concessão de uma bolsa de estudos de doutorado (processo FAPESP n . 2013/20483-6). O segundo autor agradece à FAPESP pela concessão do Auxílio à Pesquisa Jovem Pesquisador (processo FAPESP $n^{\circ}$. 2006/55835-6).

\section{REFERÊNCIAS}

AASHTO (2010) AASHTO MP19 - Standard Specification for Performance-Graded Asphalt Binder Using Multiple Stress Creep Recovery (MSCR) Test. American Association of State Highway and Transportation Officials, Washington.

Anderson, M. e J. Bukowski (2012) Using the Multiple-StressCreep-Recovery (MSCR) Test. Proceedings of the North Central Asphalt User Producer Group Meeting, Indianapolis, IN. Apresentação em PowerPoint. Disponível em: <http://enginee ring.purdue.edu/ ncaupg/Activities/2012/presentation\%202012/ Anderson\%20-\%20MSCR\%20AI\%20NCAUPG\%202012.pdf>. Acesso em: 14 out. 2014.

Anderson, R. M. (2011) Understanding the MSCR Test and its Use in the PG Asphalt Binder Specification. Asphalt Academy Webinars, Lexington, KY. Apresentação em PowerPoint. Disponível em: www.asphaltinstitute.org/dotAsset/48a2c0b0d756-4b8c-913f-e7aaf91ef03d.pdf>. Acesso em: 14 out. 2014.

ASTM (2004) ASTM D2872 - Standard Test Method for Effect of Heat and Air on a Moving Film of Asphalt (Rolling Thin-Film
Oven Test). American Society for Testing and Materials, West Conshohocken. DOI: 10.1520/D2872-04.

ASTM (2006) ASTM D5 - Standard Test Method for Penetration of Bituminous Materials. American Society for Testing and Materials, West Conshohocken. DOI: 10.1520/D0005-06.

ASTM (2006) ASTM D4402 - Standard Test Method for Viscosity Determination of Asphalt at Elevated Temperatures Using a Rotational Viscometer. American Society for Testing and Materials, West Conshohocken. DOI: 10.1520/D4402-06.

ASTM (2009) ASTM D36 - Standard Test Method for Softening Point of Bitumen (Ring-and-Ball Apparatus). American Society for Testing and Materials, West Conshohocken. DOI: 10.1520/D0036_D0036M-09.

ASTM (2010) ASTM D7405 - Standard Test Method for Multiple Stress Creep and Recovery (MSCR) of Asphalt Binder Using a Dynamic Shear Rheometer. American Society for Testing and Materials, West Conshohocken. DOI: 10.1520/D7405-10A.

Bahia, H. U (2014) Performance de Asfaltos Modificados com Polímeros. Anais do $21^{\circ}$. Encontro de Asfalto, Rio de Janeiro, RJ. Apresentação em PowerPoint.

Baumgardner, G. L (2012) Why and How of Polyphosphoric Acid Modification: An Industry Perspective. Transportation Research Circular, n. E-C160, p. 14-26.

Buncher, M. e J. A. D'Angelo (2012) Establishing a Baseline of Knowledge Through 2005 by Reviewing AI IS-220:

Polyphosphoric Acid Modification of Asphalt. Transportation Research Circular, n. E-C160, p. 12-13.

D'Angelo, J. A (2009) The Relationship of the MSCR Test to Rutting. Road Materials and Pavement Design, v. 10, supl. 1, p. 61-80. DOI: 10.1080/14680629.2009.9690236.

D'Angelo, J (2010) New High-Temperature Binder Specification Using Multistress Creep and Recovery. Transportation Research Circular, n. E-C147, p. 1-13.

D’Angelo, J. e R. Dongré (2009) Practical Use of Multiple Stress Creep and Recovery Test: Characterization of StyreneButadiene-Styrene Dispersion and Other Additives in PolymerModified Asphalt Binders. Transportation Research Record, $\mathrm{n}$. 2126, p. 73-82. DOI: 10.3141/2126-09.

D’Angelo, J.; R. Kluttz; R. Dongré; K. Stephens e L. Zanzotto (2007) Revision of the Superpave High Temperature Binder Specification: The Multiple Stress Creep Recovery Test. Journal of the Association of Asphalt Paving Technologists, v. 76, p. 123162.

Dreessen, S.; J. P. Planche e V. Gardel (2009) A New Performance Related Test Method for Rutting Prediction: MSCRT. In: Loizos, A.; M. L. Partl; P. Scarpas e I. L. Al-Qadi (eds.) Advanced Testing and Characterization of Bituminous Materials. Leiden, The Netherlands. DOI: 10.1201/9780203092989.ch93.

Fee, D.; R. Maldonado; G. Reinke e H. Romagosa (2010) Polyphosphoric Acid Modification of Asphalt. Transportation Research Record, n. 2179, p. 49-57. DOI: 10.3141/2179-06.

Gierhart, D (2013) Multiple Stress Creep Recovery (MSCR): Why it Should be Implemented. Proceedings of the Louisiana Transportation Conference, Baton Rouge, LA. Apresentação em 
PowerPoint. Disponível em:

<http://www.ltrc.lsu.edu/ltc_13/pdf/presentations/S43_Why\%20t

he $\% 20$ Multiple $\% 20$ Stress $\% 20$ Creep $\% 20$

Recovery\%20(MSCR)\%20Test\%20Should\%20be\%20Implemen ted_LTC2013.pdf>. Acesso em: 14 out. 2014.

Leite, L. F. M (1999) Estudos de Preparo e Caracterização de Asfaltos Modificados por Polímero. Tese (Doutorado em Ciência e Tecnologia de Polímeros) - Instituto de Macromoléculas Professora Eloisa B. Mano, Universidade Federal do Rio de Janeiro, Rio de Janeiro.

Lesueur, D (2009) The Colloidal Structure of Bitumen: Consequences on the Rheology and on the Mechanisms of Bitumen Modification. Advances in Colloid and Interface Science, v. 45, n. 1-2, p. 42-82. DOI: 10.1016/j.cis.2008.08.011.

King, G.; H. King; R. D. Pavlovich; A. L. Epps e P. Kandhal (1999) Additives in Asphalt. Journal of the Association of Asphalt Paving Technologists, v. 68, p. 32-69.

Martins, A. T.; L. F. M. Leite; M. C. C. Cravo; M. Chacur; P. A. Pinto e D. F. Assumpção (2011) Fluência e Relaxação sob Múltiplas Tensões (MSCR): Avaliação do Desempenho de Ligantes Asfálticos. Anais do XVI CILA - Congresso IberoLatinoamericano do Asfalto, Clube de Ideias, Rio de Janeiro, v. 1, p. 711-721.

Masson, J.-F (2008) Brief Review of the Chemistry of Polyphosphoric Acid (PPA) and Bitumen. Energy \& Fuels, v. 22, n. 4, p. 2637-2640. DOI: 10.1021/ef800120x.

Reinke, G (2010) Use of Hamburg Rut Testing Data to Validate the Use of Jnr as a Performance Parameter for High-Temperature Permanent Deformation. Transportation Research Circular, $\mathrm{n}$. E-C147, p. 14-24.

Tam, K. K (2012) Polyphosphoric Acid-Modified Asphalt Cement: Ontario Perspective. Transportation Research Circular, n. E-C160, p. 109-114.

Yildirim, Y (2007) Polymer Modified Asphalt Binders. Construction and Building Materials, v. 21, n. 1, p. 66-72. DOI: 10.1016/j.conbuildmat.2005.07.007.

Zhang, F. e J. Yu (2010) The Research for High-Performance SBR Compound Modified Asphalt. Construction and Building Materials, v. 24, n. 3, p. 410-418. DOI:

10.1016/j.conbuildmat.2009.10.003.

Zhang, J.; J. Wang; Y. Wu; W. Sun e Y. Wang (2009) Thermal Behaviour and Improved Properties of SBR and SBR/Natural Bitumen Modified Bitumens. Iranian Polymer Journal, v. 18, n. 6, p. 465-478.

Zhang, J.; Y. Wu; J. Wang; Y. Wang e Y. Wang (2007). Improved Properties of Weathered Coal and SBR/Weathered Coal Compound Modified Asphalt. Iranian Polymer Journal, v. 16, n. 4, p. 251-259. 